\title{
A Prospective Multicenter Study Evaluating Bleeding Risk after Endoscopic Ultrasound-Guided Fine Needle Aspiration in Patients Prescribed Antithrombotic Agents
}

Kazumichi Kawakubo ${ }^{1}$, Kei Yane ${ }^{2}$, Kazunori Eto ${ }^{3}$, Hirotoshi Ishiwatari ${ }^{4}$, Nobuyuki Ehira ${ }^{5}$, Shin Haba ${ }^{6,7}$, Ryusuke Matsumoto ${ }^{8}$, Keisuke Shinada ${ }^{9}$, Hiroaki Yamato ${ }^{10}$, Taiki Kudo ${ }^{7,10}$, Manabu Onodera ${ }^{6,11}$, Toshinori Okuda ${ }^{12}$, Yoko Taya-Abe ${ }^{13}$, Shuhei $K^{K a w a h a t a}{ }^{1}$, Kimitoshi Kubo ${ }^{1}$, Yoshimasa Kubota ${ }^{1}$, Masaki Kuwatani ${ }^{1}$, Hiroshi Kawakami ${ }^{1}$, Akio Katanuma ${ }^{2}$, Michihiro $\mathrm{Ono}^{4}$, Tsuyoshi Hayashi ${ }^{4}$, Minoru Uebayashi ${ }^{5}$, and Naoya Sakamoto ${ }^{1}$

${ }^{I}$ Department of Gastroenterology and Hepatology, Faculty of Medicine and Graduate School of Medicine, Hokkaido University, ${ }^{2}$ Center for Gastroenterology, Teine Keijinkai Hospital, Sapporo, ${ }^{3}$ Department of Gastroenterology, Tomakomai City Hospital, Tomakomai, ${ }^{4}$ Department of Medical Oncology and Hematology, Sapporo Medical University, Sapporo, ${ }^{5}$ Department of Gastroenterology, Japanese Red Cross Kitami Hospital, Kitami, ${ }^{6}$ Department of Gastroenterology, NTT East Sapporo Hospital, Sapporo, ${ }^{7}$ Department of Gastroenterology, Kushiro Rosai Hospital, Kushiro, ${ }^{8}$ Department of Gastroenterology, Obihiro Kosei Hospital, Obihiro, ${ }^{9}$ Department of Gastroenterology, Keiwakai Ebetsu Hospital, Ebetsu, ${ }^{10}$ Department of Gastroenterology and Hepatology, Hakodate City Hospital, Hakodate, ${ }^{11}$ Department of Gastroenterology, Abashiri Kosei Hospital, Abashiri, ${ }^{12}$ Department of Gastroenterology, Oji General Hospital, Tomakomai, and ${ }^{13}$ Department of Gastroenterology, National Hospital Organization Hokkaido Medical Center, Sapporo, Japan

See editorial on page 225.

Background/Aims: Although the risk of bleeding after endoscopic ultrasound-guided fine needle aspiration (EUS-FNA) is low, the safety of EUS-FNA in patients prescribed antithrombotic agents is unclear. Therefore, this study evaluated the incidence of bleeding after EUS-FNA in those patients. Methods: Between September 2012 and September 2015, patients who were prescribed antithrombotic agents underwent EUS-FNA at 13 institutions in Japan were prospectively enrolled in the study. The antithrombotic agents were managed according to the guidelines of the Japanese Gastrointestinal Endoscopy Society. The rate of bleeding events, thromboembolic events and other complications within 2 weeks after EUS-FNA were analyzed. Results: Of the 2,629 patients who underwent EUS-FNA during the study period, 85 (62 males; median age, 74 years) patients were included in this stduy. Two patients (2.4\%; 95\% confidence interval [Cl], 0.6\% to $8.3 \%)$ experienced bleeding events. One patient required surgical intervention for hemothorax 5 hours after EUS-FNA, and the other experienced melena 8 days after EUS-FNA and required red blood cell transfusions. No thromboembolic events occurred $(0 \% ; 95 \% \mathrm{Cl}, 0.0 \%$ to $4.4 \%)$. Three patients (3.5\%; 95\% Cl, 1.2\% to $10.0 \%$ ) experienced peri-puncture abscess formation. Conclusions: The rate of bleeding after EUS-FNA in patients prescribed antithrombotic agents might be considerable. (Gut Liver 2018;12:353-359)

Key Words: Endoscopic ultrasound-guided fine needle aspiration; Hemorrhage; Fibrinolytic agents

\section{INTRODUCTION}

Endoscopic ultrasound-guided fine needle aspiration (EUSFNA) is the most reliable method for the histological diagnosis of pancreatic and gastrointestinal submucosal tumors, as well as mediastinal and abdominal lymphadenopathies. ${ }^{1-3}$ The rate of overall EUS-FNA specific morbidity has been reported to be 0.98\%. ${ }^{4}$ Major adverse events after EUS-FNA include postprocedure pain, pancreatitis, bleeding and fever.

The number of patients taking antithrombotic agents to prevent thromboembolic events is increasing. ${ }^{5,6}$ For example, $10.5 \%$ of patients in Japan undergoing endoscopic mucosal biopsy were taking antithrombotic agents. ${ }^{7}$ Guidelines have recommended that antithrombotic agents be managed in pa-

Correspondence to: Kazumichi Kawakubo

Department of Gastroenterology and Hepatology, Faculty of Medicine and Graduate School of Medicine, Hokkaido University, Kita 15 Nishi 7, Kitaku, Sapporo 060-8638, Japan

Tel: +81-117161161, Fax: +81-117067867, E-mail: kkawakubo-gi@umin.ac.jp

Received on July 5, 2017. Revised on September 8, 2017. Accepted on September 20, 2017. Published online February 8, 2018 pISSN 1976-2283 eISSN 2005-1212 https://doi.org/10.5009/gnl17293

The manuscript was presented as part at Digestive Disease Week 2016, at San Diego, USA (http://www.giejournal.org/article/S0016-5107(16)00510-1/ abstract).

(a) This is an Open Access article distributed under the terms of the Creative Commons Attribution Non-Commercial License (http://creativecommons.org/licenses/by-nc/4.0) which permits unrestricted non-commercial use, distribution, and reproduction in any medium, provided the original work is properly cited. 
tients undergoing endoscopic procedures, based on a balance between the risk of bleeding associated with the procedure and the risk of thrombotic events associated with the underlying condition. Guidelines of the American Society of Gastrointestinal Endoscopy and the Japanese Society of Gastrointestinal Endoscopy (JSGE) have regarded EUS-FNA as having a high risk of bleeding, whereas the guidelines of the European Society of Gastrointestinal Endoscopy regard this procedure as having a low risk of bleeding. ${ }^{8-10}$ Only one study to date has evaluated the safety of EUS-FNA in patients taking aspirin, but that was a single-center study performed in a relatively small number of patients. ${ }^{11}$ We hypothesized that bleeding risk after EUS-FNA would be low among patients taking antithrombotic agents, but there have been no reports about the safety of EUS-FNA in patients taking other antiplatelet agents and/or anticoagulants. This study therefore analyzed the safety of EUS-FNA among patients taking antithrombotic agents, including antiplatelet and anticoagulant agents.

\section{MATERIALS AND METHODS}

\section{Study design}

This prospective, multicenter study enrolled patients taking antithrombotic agents who underwent EUS-FNA in accordance with Japanese guidelines between September 2012 and September 2015 at 13 institutions in Japan. Indications for EUS-FNA were basically determined by European Society of Gastrointestinal Endoscopy guidelines. ${ }^{12}$ This study was approved by the Institutional Review Board of each hospital and registered at www.ClinicalTrials.gov (NCT01798654). All authors had access to the study data and reviewed and approved the final manuscript.

\section{Patients}

Patients were included if they were older than 20 years, were at high risk of thrombotic events according to the guidelines of the JGES, ${ }^{8}$ and provided written informed consent to be enrolled in this study. Patients were excluded if they had pancreatic cystic lesions, a platelet count $<50,000 / \mathrm{m}^{3}$, an American Society of Anesthesiologists Physical Status Classification System score $>3$ or an Eastern Cooperative Oncology Group performance status of 4. Written informed consent was obtained from each patient prior to EUS-FNA.

\section{Management of antithrombotic agents}

Before EUS-FNA, antithrombotic agents were managed according to modified JSGE guidelines. ${ }^{8}$ Aspirin was continued throughout the procedure. Thienopyridines were discontinued for 5 days and replaced with aspirin. Antiplatelet agents other than thienopyridines were discontinued for 1 day. Warfarin was discontinued for 3 days and non-vitamin $\mathrm{K}$ antagonist oral anticoagulants (NOAC) were discontinued for 2 days, with both replaced by intravenous heparin (10,000 to 20,000 units/day). Intravenous heparin was stopped 3 hours before EUS-FNA, restarted 1 day later and maintained until the international normalized ratio (INR) reached its therapeutic range (1.5 to 2.5). All antithrombotics were resumed 1 day after EUS-FNA. In patients taking NOACs, however, heparin was not re-administered after EUS-FNA.

\section{EUS-FNA procedure and follow-up}

EUS-FNA was performed in a standard manner, using a standard EUS-FNA needle and negative pressure suction, in an inpatient setting by endoscopists with experience performing more than 50 EUS-FNAs. The selection of needle size and the number of punctures was at the discretion of each endoscopist. After EUS-FNA, the patient was discharged and followed-up for 2 weeks.

\section{Outcome measurements}

The primary outcome measure was the rate of bleeding complication within 2 weeks. Patients with melena or other symptoms suggesting bleeding events underwent upper gastrointestinal endoscopy or other imaging examinations to confirm the bleeding site. Otherwise, patients were interviewed at the outpatient clinic or by telephone about the presence of melena or other symptoms 2 weeks after EUS-FNA, thus eliminating attrition bias. The grading and severity of bleeding complications were defined by the American Society of Gastrointestinal Endoscopy guidelines. ${ }^{13}$ Secondary outcome measures included the rates of thromboembolic and other adverse events and the histopathological diagnosis of EUS-FNA.

\section{Sample size calculation}

The incidence of bleeding after EUS-FNA was estimated at $1.0 \% .{ }^{4}$ Calculations showed that a sample size of 300 patients was required to detect a bleeding rate of $1 \%$, in accordance with the rule of three.

\section{Statistical analysis}

Categorical variables were described as proportions and continuous variables as medians and ranges. The rates of bleeding complications, thromboembolic events and other complications were calculated, along with their 95\% confidence intervals (CIs). All statistical analyses were performed using JMP version 11 (SAS Institute Inc., Cary, NC, USA).

\section{RESULTS}

\section{Patient characteristics}

During the study period, 2,629 patients underwent EUS-FNA for solid lesions; among these, 120 patients (4.6\%) were tak- 
ing antithrombotic agents (Fig. 1). Eighty-seven patients were deemed at high risk for thrombotic events according to JSGE guidelines and included in this study. After excluding two patients, one with a high American Society of Anesthesiologists score and the other who refused consent, 85 patients were included in this study (Table 1). Their median age was 74 years (range, 40 to 85 years) and 62 patients $(72.9 \%)$ were male. Median platelet count was $195 \times 10^{3} / \mathrm{mm}^{3}$ (range, 57 to $370 \times 10^{3}$ ) $\mathrm{mm}^{3}$ ), and median INR before EUS-FNA was 1.09 (range, 0.90 to 1.66). Numbers of antithrombotic agents taken by patients are shown in Table 1 and the reasons for antithrombotic agents in Table 2. Fifty-one patients (60.0\%) were each taking a single antithrombotic agent, whereas 28 (32.9\%) and six (7.1\%) patients were taking two and three antithrombotic agents, respectively.

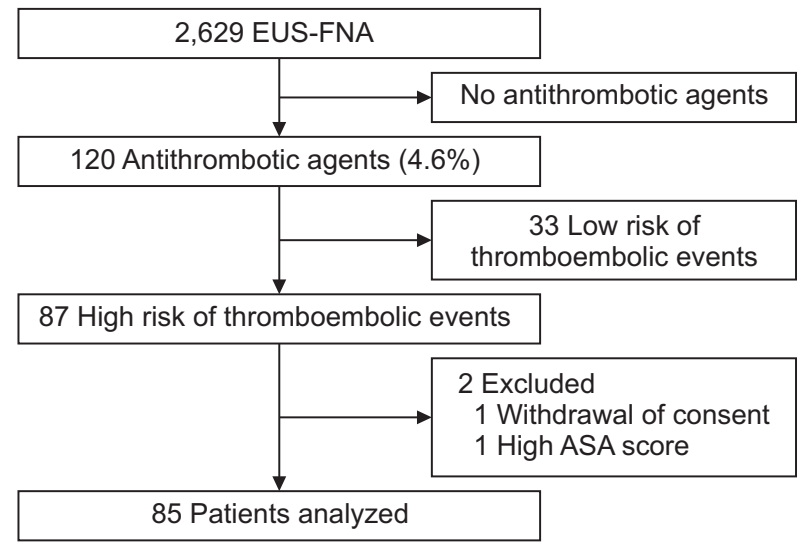

Fig. 1. Flow chart of this study.

EUS-FNA, endoscopic ultrasound-guided fine needle aspiration; ASA, American Society of Anesthesiologists.

\section{EUS-FNA procedures}

The target lesions in the 85 patients included pancreatic tumors in 54 (63.5\%), lymph nodes in 11 (12.9\%), gastrointestinal submucosal tumors in 10 (11.8\%), bile ducts in five (5.9\%) and others in five (5.9\%). The median target lesion diameter was $24.2 \mathrm{~mm}$ (range, 6.0 to $130.0 \mathrm{~mm}$ ). Gastrointestinal puncture sites included the stomach in 43 patients (50.6\%), the duodenum in 38 (44.7\%), the esophagus in three (3.5\%) and the small intestine in one (1.2\%). Needle size was 19 gauge in 11 patients

Table 1. Patient Characteristics and Antithrombotic Agents Administered to Patients Prior to EUS-FNA

\begin{tabular}{lc}
\hline \multicolumn{1}{c}{ Variable } & Value \\
\hline Age, yr & $74(40-85)$ \\
Male sex & $62(72.9)$ \\
Platelets, $\times 10^{4} / \mathrm{mm}^{3}$ & $19.5(5.7-37.0)$ \\
PT-INR & $1.09(0.90-1.66)$ \\
Antithrombotic agents & \\
Antiplatelets & \\
$\quad$ Aspirin & 43 \\
Thienopyridine & 30 \\
Cilostazol & 8 \\
Others & 4 \\
Anticoagulants & \\
Warfarin & 25 \\
NOAC & 14 \\
No. of antithrombotic agents & \\
1/2/3 & $51 / 28 / 6$ \\
\hline
\end{tabular}

Data are presented as median (range) or number (\%).

EUS-FNA, endoscopic ultrasound-guided fine needle aspiration; PTINR, prothrombin time-international normalized ratio; NOAC, nonvitamin $\mathrm{K}$ antagonist oral anticoagulants.

Table 2. Reasons for Antithrombotic Agent Prescriptions

Two months after insertion of a bare metal stent into the coronary artery

Twelve months after insertion of a drug-eluting stent into the coronary artery

Ischemic stroke or transient ischemic attack with $>50 \%$ stenosis of major intracranial arteries

12

Recent ischemic stroke or transient ischemic attack

8

Obstructive peripheral artery disease $\geq$ Fontaine grade 3 (rest pain)

Ultrasonic examination of carotid arteries and magnetic resonance angiography of the head and neck region where withdrawal is

considered high risk of thromboembolism

History of cardiogenic brain embolism

Atrial fibrillation accompanying valvular heart disease

Atrial fibrillation without valvular heart disease but with high risk of stroke

Following mechanical mitral valve replacement

History of thromboembolism following mechanical valve replacement 
Table 3. Histological Diagnosis of Endoscopic Ultrasound-Guided Fine Needle Aspiration

\begin{tabular}{lc}
\hline Adenocarcinoma & 44 \\
Adenosquamous carcinoma & 1 \\
Squamous cell carcinoma & 3 \\
Clear cell carcinoma & 2 \\
Neuroendocrine tumor & 4 \\
Undifferentiated carcinoma & 1 \\
Small cell carcinoma & 1 \\
Gastrointestinal stromal tumor & 5 \\
Leiomyoma & 1 \\
Lymphoma & 2 \\
Paraganglioma & 1 \\
Solid pseudopapillary neoplasm & 1 \\
Atypical epithelial cells & 4 \\
Nonneoplastic tissue & 13 \\
Insufficient materials & 1 \\
Not analyzed & 1 \\
\hline
\end{tabular}

(12.9\%), 22 gauge in 65 (76.5\%) and 25 gauge in nine (10.6\%). The median number of punctures was 3 (range, 1 to 7). Rapid on-site evaluation was performed in 68 patients (80.0\%). Based on the results of EUS-FNA, 66 patients (77.6\%) were histologically diagnosed with neoplastic diseases (Table 3).

\section{Bleeding complications}

Two patients $(2.4 \%$; $95 \%$ CI, $0.6 \%$ to $8.3 \%)$ experienced bleeding complications. The first patient was a 72-year old male who was taking aspirin and thienopyridine because of a recent ischemic stroke. He discontinued thienopyridine for 5 days and underwent EUS-FNA of a submucosal tumor at the gastric fornix using a 19-gauge needle with two needle passes and was histologically diagnosed with a gastrointestinal stromal tumor. Five hours after EUS-FNA, he developed dyspnea and hypoxia. Chest-computed tomography showed left hemothorax, and he underwent emergency video-assisted thoracoscopic surgery for the confirmation of hemostasis. He was discharged 2 weeks after surgery. The second patient was a 62-year-old male, who was taking warfarin because of a mechanical valve replacement. Three days before EUS-FNA, warfarin was discontinued and replaced by intravenous heparin. He underwent EUS-FNA of a swollen para-aortic lymph node using a 25-gauge needle. Although discharged without complications, he presented with melena 8 days after EUS-FNA. Upper gastrointestinal endoscopy showed a duodenal ulcer, but no bleeding at the EUS-FNA puncture site in the stomach. He was transfused with two units of red blood cells. The rates of bleeding complications according to needle size were 9.1\% (1/11), 0\% (0/65), and 11.1\% (1/9) for 19-, 22-, and 25-gauge needles, respectively. There were no bleeding events in the 17 patients who required only a single dose of aspirin or cilostazol.

\section{Other complications}

No patient experienced thromboembolic events and there were no procedure related deaths $(0 \% ; 95 \% \mathrm{CI}, 0.0 \%$ to $4.4 \%)$. Three patients (3.5\%; 95\% CI, 1.2\% to $10.0 \%$ ) experienced other complications, with all showing abscess formation around the puncture site. One patient required endoscopic ultrasoundguided abscess drainage, whereas the other two were managed conservatively.

\section{DISCUSSION}

Our study demonstrated, for the first time, that the rate of bleeding complications after EUS-FNA for patients taking antithrombotic agents was $2.4 \%$. In the rule of three, the upper limit of the $95 \% \mathrm{CI}$ of the probability of an event is calculated as 3 divided by the number of procedures if no event occurred in a finite number of procedures. ${ }^{14}$ The rate of bleeding in this study exceeded the estimated upper limit of the 95\% CI (1\%). From this point of view, the rate of bleeding events in patients with antithrombotic agents was not as low as $1 \%$, and the relatively high rate of bleeding complications in our study should not be generalized.

A previous meta-analysis showed that the rate of bleeding complications after EUS-FNA was $0.13 \%,{ }^{4}$ and assessments of the Japanese national database reported that the rates of severe bleeding after EUS-FNA in patients with gastrointestinal submucosal tumors and pancreatic tumors were $0.44 \%$ and $0.23 \%$, respectively. ${ }^{15,16} \mathrm{~A}$ recent retrospective study reported that EUSFNA-related bleeding was as low in patients with antithrombotic agents as in those without them. ${ }^{17}$ The retrospective nature of the present study, however, did not allow estimation of postoperative bleeding complications. In agreement with an earlier study, we found that $4.6 \%$ of patients undergoing EUS-FNA were taking antithrombotic agents. ${ }^{7}$ The higher rate of bleeding complications in our study of patients taking antithrombotic agents was likely due to their higher risk for bleeding complications after invasive endoscopic procedures and the patients' comorbidities that required antithrombotic agents. ${ }^{18,19}$

The efficacy of perioperative heparin bridging in preventing thromboembolic events is unclear. ${ }^{20}$ One of the patients in our study who was taking warfarin experienced a bleeding complication, but this was considered related to a comorbidity rather than the EUS-FNA procedure itself. A recent large, randomized controlled trial reported that heparin bridging increased the risk of major bleeding without reducing the risk of thromboembolism. ${ }^{21}$ The incidence of bleeding complications following endoscopic mucosal biopsy in patients treated with antithrombotic agents was reported to be low, even without discontinuation of 
these agents. ${ }^{22}$ The lack of thromboembolic events in our study suggested that warfarin discontinuation with heparin bridging would not be necessary prior to EUS-FNA in patients taking warfarin. Furthermore, NOACs have been shown more effective and safe than warfarin in preventing thromboembolic events. ${ }^{23,24}$ Because few patients in our study were taking NOACs, the safety of EUS-FNA for those patients remains to be determined.

We also found that one patient taking both aspirin and thienopyridine, discontinuing the latter before EUS-FNA, experienced a severe bleeding complication. This was consistent with findings showing that dual antiplatelet treatment was a risk factor for bleeding complications after gastric endoscopy. ${ }^{25}$ Because stent thrombosis is a life-threatening event, dual antiplatelet therapy for at least 1 year has been recommended after drugeluting stent placement. ${ }^{26,27}$ However, novel drug-eluting stents have a low risk of restenosis following a short duration of dual antiplatelet treatment, suggesting that the number of patients will likely decrease in the near future. ${ }^{28,29}$ Nevertheless, caution before EUS-FNA is necessary for those patients.

In our study, there were no bleeding events in any of the patients who took aspirin or cilostazol without interruption. This finding was consistent with that of a previous study by Inoue et al. ${ }^{17}$ Therefore, use of EUS-FNA may be feasible with continued aspirin or cilostazol.

Another concern in the management of antithrombotic agents for endoscopic procedures is that a thromboembolic event may occur after the discontinuation of antithrombotic agents. ${ }^{30}$ Thromboembolic events may be more harmful than bleeding events and may even be fatal. ${ }^{31}$ None of the patients in our study, however, experienced a thromboembolic event. Because bleeding events could be successfully managed without any sequelae or mortality, EUS-FNA may be feasible without discontinuing antithrombotic agents.

We observed three patients with abscess formation around the puncture site. The reason for relatively high incidence of this complication was not clear, but it might be possible that the enrolled patients had comorbid diseases requiring antithrombotic agents, so the healing process may have been compromised.

This study had several limitations, including the relatively small number of patients, making its statistical power low. In addition, this was not a comparative study because the huge number of patients is mandatory to evaluate the rate of rare events. Instead, we calculated the sample size in accordance with the rule of three. EUS-FNA procedures may have differed among the participating institutions; therefore performance bias could not be excluded. Furthermore, patients in this study were taking various antithrombotic agents, making comparisons difficult; and only Japanese patients were included, making it difficult to generalize these results among different ethnic groups. We excluded patients with cystic lesions (who were more likely to develop bleeding complications) than those with solid lesions, so we might have underestimated the overall rate of bleeding complications. Finally, we could not determine whether bleeding was due to the antithrombotic agents themselves or the comorbidity requiring those agents.

The rate of bleeding complications after EUS-FNA for patients taking antithrombotic agents might be considerable, regardless of whether the bleeding was attributed to EUS-FNA itself. Endoscopists should evaluate the risks of both thromboembolic events and bleeding complications before performing EUS-FNA on patients being treated with antithrombotic agents. A large prospective study is necessary to develop new guidelines for the management of patients with antithrombotic agents who undergo EUS-FNA.

\section{CONFLICTS OF INTEREST}

No potential conflict of interest relevant to this article was reported.

\section{ACKNOWLEDGEMENTS}

This study was supported by a grant from the Japanese Foundation for Research and Promotion of Endoscopy. The founder had no role in the study design, nor collection, analysis, or interpretation of the data.

We express our greatest appreciation to Dr. Hiroaki Iijima for statistical support.

Author contributions: Kazumichi Kawakubo contributed to study design; acquisition, analysis and interpretation of data; and drafting of the manuscript. K.Y., K.E., H.I., N.E., S.H. R.M., K.S., H.Y., T.K., M.O., T.O., Y.T.A., S.K., Kimitoshi Kubo, Y.K., M.O., and M.U. contributed to study design; and acquisition, analysis and interpretation of data. M.K., H.K., T.H., A.K., and N.S. contributed to study design; acquisition, analysis and interpretation of the data; and critical revision of the manuscript.

\section{REFERENCES}

1. Hewitt MJ, McPhail MJ, Possamai L, Dhar A, Vlavianos P, Monahan KJ. EUS-guided FNA for diagnosis of solid pancreatic neoplasms: a meta-analysis. Gastrointest Endosc 2012;75:319-331.

2. Polkowski M, Larghi A, Weynand B, et al. Learning, techniques, and complications of endoscopic ultrasound (EUS)-guided sampling in gastroenterology: European Society of Gastrointestinal Endoscopy (ESGE) technical guideline. Endoscopy 2012;44:190206.

3. Yasuda I, Goto N, Tsurumi H, et al. Endoscopic ultrasound-guided fine needle aspiration biopsy for diagnosis of lymphoproliferative disorders: feasibility of immunohistological, flow cytometric, and cytogenetic assessments. Am J Gastroenterol 2012;107:397-404.

4. Wang KX, Ben QW, Jin ZD, et al. Assessment of morbidity and 
mortality associated with EUS-guided FNA: a systematic review. Gastrointest Endosc 2011;73:283-290.

5. Furie KL, Goldstein LB, Albers GW, et al. Oral antithrombotic agents for the prevention of stroke in nonvalvular atrial fibrillation: a science advisory for healthcare professionals from the American Heart Association/American Stroke Association. Stroke 2012;43:3442-3453.

6. Grines CL, Bonow RO, Casey DE Jr, et al. Prevention of premature discontinuation of dual antiplatelet therapy in patients with coronary artery stents: a science advisory from the American Heart Association, American College of Cardiology, Society for Cardiovascular Angiography and Interventions, American College of Surgeons, and American Dental Association, with representation from the American College of Physicians. Circulation 2007;115:813-818.

7. Ara N, Iijima K, Maejima R, et al. Prospective analysis of risk for bleeding after endoscopic biopsy without cessation of antithrombotics in Japan. Dig Endosc 2015;27:458-464.

8. Fujimoto K, Fujishiro M, Kato M, et al. Guidelines for gastroenterological endoscopy in patients undergoing antithrombotic treatment. Dig Endosc 2014;26:1-14.

9. ASGE Standards of Practice Committee, Anderson MA, BenMenachem T, et al. Management of antithrombotic agents for endoscopic procedures. Gastrointest Endosc 2009;70:1060-1070.

10. Boustière C, Veitch A, Vanbiervliet G, et al. Endoscopy and antiplatelet agents. European Society of Gastrointestinal Endoscopy (ESGE) Guideline. Endoscopy 2011;43:445-461.

11. Kien-Fong Vu C, Chang F, Doig L, Meenan J. A prospective control study of the safety and cellular yield of EUS-guided FNA or Trucut biopsy in patients taking aspirin, nonsteroidal anti-inflammatory drugs, or prophylactic low molecular weight heparin. Gastrointest Endosc 2006;63:808-813.

12. Dumonceau JM, Polkowski M, Larghi A, et al. Indications, results, and clinical impact of endoscopic ultrasound (EUS)-guided sampling in gastroenterology: European Society of Gastrointestinal Endoscopy (ESGE) clinical guideline. Endoscopy 2011;43:897-912.

13. Cotton PB, Eisen GM, Aabakken L, et al. A lexicon for endoscopic adverse events: report of an ASGE workshop. Gastrointest Endosc 2010;71:446-454.

14. Eypasch E, Lefering R, Kum CK, Troidl H. Probability of adverse events that have not yet occurred: a statistical reminder. BMJ 1995;311:619-620.

15. Hamada T, Yasunaga H, Nakai Y, et al. Rarity of severe bleeding and perforation in endoscopic ultrasound-guided fine needle aspiration for submucosal tumors. Dig Dis Sci 2013;58:2634-2638.

16. Hamada T, Yasunaga H, Nakai Y, et al. Severe bleeding and perforation are rare complications of endoscopic ultrasound-guided fine needle aspiration for pancreatic masses: an analysis of 3,090 patients from 212 hospitals. Gut Liver 2014;8:215-218.

17. Inoue T, Okumura F, Sano H, et al. Bleeding risk of endoscopic ultrasound-guided fine-needle aspiration in patients undergoing antithrombotic therapy. Dig Endosc 2017;29:91-96.

18. Koh R, Hirasawa K, Yahara S, et al. Antithrombotic drugs are risk factors for delayed postoperative bleeding after endoscopic submucosal dissection for gastric neoplasms. Gastrointest Endosc 2013;78:476-483.

19. Hamada T, Yasunaga H, Nakai Y, et al. Bleeding after endoscopic sphincterotomy or papillary balloon dilation among users of antithrombotic agents. Endoscopy 2015;47:997-1004.

20. Matsumoto M, Mabe K, Tsuda M, et al. Multicenter study on hemorrhagic risk of heparin bridging therapy for periendoscopic thromboprophylaxis. BMC Gastroenterol 2015;15:89.

21. Douketis JD, Spyropoulos AC, Kaatz S, et al. Perioperative bridging anticoagulation in patients with atrial fibrillation. N Engl J Med 2015;373:823-833.

22. Ono S, Fujishiro M, Kodashima S, et al. Evaluation of safety of endoscopic biopsy without cessation of antithrombotic agents in Japan. J Gastroenterol 2012;47:770-774.

23. Granger CB, Alexander JH, McMurray JJ, et al. Apixaban versus warfarin in patients with atrial fibrillation. $\mathrm{N}$ Engl J Med 2011;365:981-992.

24. Schulman S, Kearon C, Kakkar AK, et al. Dabigatran versus warfarin in the treatment of acute venous thromboembolism. N Engl $\mathrm{J}$ Med 2009;361:2342-2352.

25. Ono S, Fujishiro M, Yoshida N, et al. Thienopyridine derivatives as risk factors for bleeding following high risk endoscopic treatments: Safe Treatment on Antiplatelets (STRAP) study. Endoscopy 2015;47:632-637.

26. Windecker S, Kolh P, Alfonso F, et al. 2014 ESC/EACTS Guidelines on myocardial revascularization: The Task Force on Myocardial Revascularization of the European Society of Cardiology (ESC) and the European Association for Cardio-Thoracic Surgery (EACTS) developed with the special contribution of the European Association of Percutaneous Cardiovascular Interventions (EAPCI). Eur Heart J 2014;35:2541-2619.

27. Levine GN, Bates ER, Blankenship JC, et al. 2011 ACCF/AHA/ SCAI guideline for percutaneous coronary intervention: a report of the American College of Cardiology Foundation/American Heart Association Task Force on practice guidelines and the Society for Cardiovascular Angiography and Interventions. Circulation 2011;124:e574-e651.

28. Colombo A, Chieffo A, Frasheri A, et al. Second-generation drugeluting stent implantation followed by 6- versus 12-month dual antiplatelet therapy: the SECURITY randomized clinical trial. J Am Coll Cardiol 2014;64:2086-2097.

29. Gilard M, Barragan P, Noryani AA, et al. 6- versus 24-month dual antiplatelet therapy after implantation of drug-eluting stents in patients nonresistant to aspirin: the randomized, multicenter ITALIC trial. J Am Coll Cardiol 2015;65:777-786.

30. Fujishiro M, Oda I, Yamamoto Y, et al. Multi-center survey regard- 
ing the management of anticoagulation and antiplatelet therapy for endoscopic procedures in Japan. J Gastroenterol Hepatol 2009;24:214-218.
31. Claessen BE, Henriques JP, Jaffer FA, Mehran R, Piek JJ, Dangas GD. Stent thrombosis: a clinical perspective. JACC Cardiovasc Interv 2014;7:1081-1092. 